\title{
Loss of Masticatory Function Affects Growth and Development of the Mandibular Condyle in Rats
}

\section{Kei Ogawa, Yuri Kiguchi, Seiko Yamamoto-Nemoto, Norimitsu Hirai, Kanako Sawamoto, Takehiko Shimizu}

Department of Pediatric Dentistry, Nihon University School of Dentistry at Matsudo, Chiba, Japan

Email: ogawa.kei@nihon-u.ac.jp

How to cite this paper: Ogawa, K., Kiguchi, Y., Yamamoto-Nemoto, S., Hirai, N., Sawamoto, K. and Shimizu, T. (2016) Loss of Masticatory Function Affects Growth and Development of the Mandibular Condyle in Rats. Open Journal of Stomatology, 6, 261273.

http://dx.doi.org/10.4236/ojst.2016.612032

Received: October 17, 2016

Accepted: December 11, 2016

Published: December 14, 2016

Copyright $\odot 2016$ by authors and Scientific Research Publishing Inc. This work is licensed under the Creative Commons Attribution International License (CC BY 4.0).

http://creativecommons.org/licenses/by/4.0/

\begin{abstract}
The effects of childhood masticatory function loss and soft foods on the mandibular condyle have been the subject of much research. However, the corresponding bone turnover is not fully understood. The purpose of the present study was to clarify the effects of a lack of teeth and a soft food diet during the growth period on bone turnover in the mandibular condyle. We divided 3-week-old Wistar rats into the following three groups: 1) Extraction group: The maxillary molars were extracted at the age of 4 weeks, and animals were fed powdered standard feed. 2) Powder group: Animals were fed powdered standard feed without tooth extraction. 3) Control group: Animals were fed solid standard feed without tooth extraction.Non-decalcified thin-slice specimens of sagittal sections of the mandibular condyle were obtained at the age of 20 weeks for histological analysis. We used micro-CT analysis and bone histomorphometry to measure bone volume (BV), bone mineral content (BMC), bone mineral density (BMD), bone microstructure, bone resorption, and osteogenesis in the mandibular condyle, and we compared the results among groups. In the extraction and the powder groups, we found deformation and disruption of the arrangement of chondrocytes, coagulation of chondrocytes, and duplication of the tidemark in the cartilage. We also found an increase in multinuclear osteoclasts in the cancellous bone. We found a reduction in BV, BMC, and BMD in the extraction and powder groups compared to the control group, as well as a reduction of bone volume, a lowering of osteogenesis parameters, and an increase in bone resorption parameters in the secondary cancellous bone. These results suggest that a lack of teeth and a soft food diet during the growth period cause a decline in bone microstructure, a decrease in osteogenesis, and an increase in bone resorption.
\end{abstract}

\section{Keywords}

Rat, Mandibular Condyle, Powder Diet, Extraction of Molars, Bone

Histomorphometry 


\section{Introduction}

Pediatric dental clinics often encounter cases of dental caries or injury leading to tooth loss as well as cases of congenital lack of teeth. Additionally, the relatively recent increase in artificial foods and the corresponding increase in soft foods have given rise to a tendency for children to prefer soft foods rather than hard foods. There have been many animal experiments reporting that tooth extraction and soft foods detrimentally affect the development of the facial cranium, mandibular bone, and masticatory muscles [1]-[6]. We have previously reported that raising rats with extraction of the maxillary molars and powdered feed reduces mandibular volume, bone density, mandibular height, and mandibular condyle thickness [7] [8]. However, the mandibular condyle shows particularly active growth during the postnatal growth of the mandible. In particular, the cartilage of the mandibular condyle functions as growth cartilage during the growth period and causes an increase in bone growth toward the rear and top of the neonatal mandibular condyle. Disruption of the arrangement of cell layers of the cartilage of the mandibular condyle and changes in cell layer thickness have been reported as effects of mandibular functional loss, malocclusion, and a soft food diet [9] [10] [11] [12] [13]. However, most of these reports used tissue observations and histochemical explorations.

Therefore, the purpose of the present study was to clarify the effects of using powdered feed to raise rats whose maxillary molars have been extracted during the growth period on the growth of the mandibular condyle. We used micro-CT to measure the bone volume (BV), the bone mineral content (BMC), and the bone mineral density (BMD) of the mandibular condyle. We also prepared non-decalcified thin-slice specimens and performed bone histomorphometry on the cartilage and the cancellous bone of the mandibular condyle in order to investigate bone turnover at both the cell level and the tissue level.

\section{Materials and Methods}

\subsection{Experimental Animals}

This study was approved by the Ethics Committee of the Nihon University School of Dentistry at Matsudo (animal experiment approval number: AP13MD020). We purchased three-week-old Wistar rats (21 males) from Sankyo Lab Service Co., Ltd., and randomly divided them into the following three groups (seven rats each): 1) Extraction group: When the rats were 4 weeks old, we intraperitoneally administered thiamylal sodium (15 mg/kg; Kyorin Pharmaceutical Co., Ltd., Tokyo, Japan) and used a spoon excavator under general anesthesia to dislocate and extract all the maxillary molars on both sides. After the extraction, we raised the rats on powdered standard feed (MF, Oriental Yeast Co., Ltd, Tokyo, Japan; Table 1. 2) Powder group: These rats were raised on powdered standard feed without tooth extraction. 3) Control group: These rats were raised on solid standard feed without tooth extraction.

Animals were raised in the rat facility at the Laboratory Animal Research Center of 
Table 1. The compositions of the powdered standard feed and solid standard feed.

\begin{tabular}{cc}
\hline Composition & Content \\
\hline Moisture (g) & 7.9 \\
Protein (g) & 23.1 \\
Lipid (g) & 5.1 \\
Ash (g) & 5.8 \\
Fiber (g) & 2.8 \\
Nitrogen free extract (g) & 55.3 \\
Calorie (kcal) & 359 \\
\hline
\end{tabular}

our university with a room temperature of $24^{\circ} \mathrm{C} \pm 1^{\circ} \mathrm{C}$; a constant humidity level of $65 \% \pm 5 \%$; and a 12 -hour light/dark cycle. All rats were given distilled water to drink and had free access to feed and water.

Tetracycline (20 mg/kg; Sigma-Aldrich, St. Louis, MO, USA) and calcein $(10 \mathrm{mg} / \mathrm{kg}$; Sigma-Aldrich) were subcutaneously injected seven days and one day before euthanasia, respectively, in order to double-label the bones. At the age of twenty weeks, the rats were euthanized with carbon dioxide gas, and mandibles on both sides were extracted.

\subsection{Measurement of Body Weight}

Rats' body weights were measured weekly from the age of three weeks until the age of twenty weeks.

\subsection{Micro-CT Analysis}

We imaged the extracted right mandibular condyle using micro-CT (R_mCT2, Rigaku, Tokyo, Japan). The imaging conditions were as follows: tube voltage, $90 \mathrm{kV}$; tube current, $160 \mu \mathrm{A}$; magnification, $2 \times$; measurement time, 3 minutes; Field of View, $3 \mathrm{~mm}$. Slice images were constructed in three dimensions using 3-D construction analysis software (TRI/3D-BON, Ratoc System Engineering Co, Tokyo, Japan). We measured the $\mathrm{BV}, \mathrm{BMC}$, and $\mathrm{BMD}$ of the mandibular condyle in a region $4 \mathrm{~mm}$ from the tip of the mandibular condyle.

\subsection{Histological Observation}

We extracted the left mandibular condyle of each rat and fixed it in $70 \%$ ethanol. We discarded the soft tissue and then immersed the bone for four days in Villanueva Bone Stain without decalcifying. After dehydrating with ethanol, we wrapped the condyle with methyl methacrylate resin. We cut sagittal sections of the mandibular condyle to a thickness of $5 \mu \mathrm{m}$ using a microtome and prepared non-decalcified thin-slice specimens for histological observation.

\subsection{Bone Histomorphometry of Cartilage}

We measured the area of each chondrocyte layer and the number of cells in each of the cell layers in the central portion of the sagittal cross-sections (Figure 1(a)). Following 

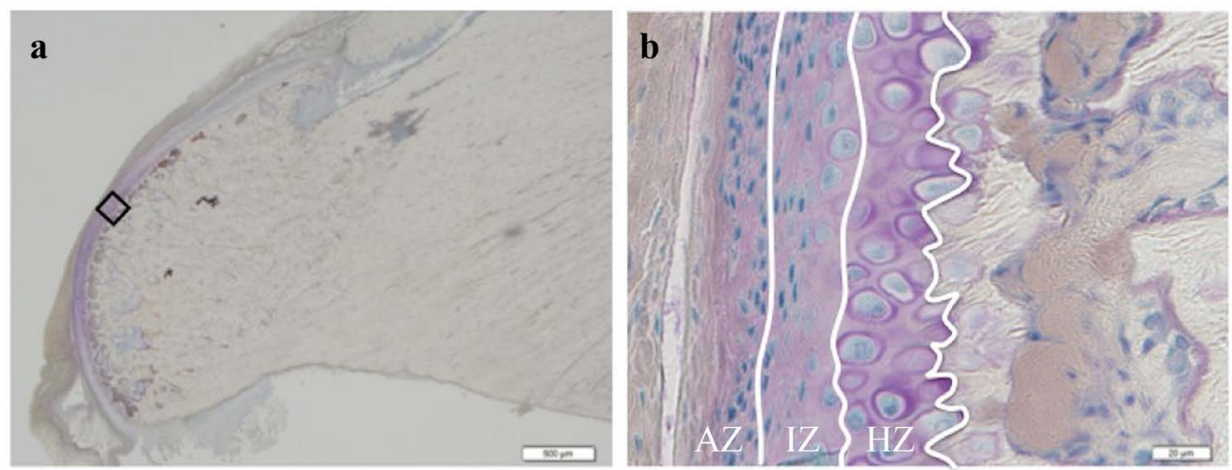

Figure 1. Measurement method for the cartilage of the mandibular condyle. (a) The square shows the region where the areas of the chondrocyte layers and the numbers of cells in each cell layer were measured (square width, $125 \mu \mathrm{m}$ ); (b) Following Blackwood [14], we categorized the cartilage layers into the articular zone (AZ), the intermediate zone (IZ), and the hypertrophic zone (HZ).

Blackwood [14], we classified the cartilage layers as articular zone (AZ), intermediate zone (IZ), or hypertrophic zone (HZ; Figure 1(b)).

\subsection{Bone Histomorphometry of Cancellous Bone}

In order to measure the mandibular condyle, a region of 418,208 to $716,928 \mu \mathrm{m}^{2}$ was selected in the secondary cancellous bone of each specimen. The following parameters were measured: bone volume/tissue volume (BV/TV), trabecular thickness (Tb.Th), osteoid volume/bone volume (OV/BV), osteoblast number/bone surface (N.Ob/BS), eroded surface/bone surface (ES/BS), osteoclast number/bone surface (N.Oc/BS), and bone formation rate/bone surface (BFR/BS). For N.Oc, mononuclear osteoclasts (N. Mo.Oc) and multinuclear osteoclasts (N.Mu.Oc) were measured separately.

\subsection{Statistical Analysis}

All statistical analyses were performed using SPSS (IBM Corp., Armonk, NY). All data are expressed as the mean \pm SD. ANOVA was used to determine differences between multiple groups, while differences between individual groups were determined by $\mathrm{Tu}$ key's test. A p value equal to or less than 0.05 was considered to be statistically significant.

\section{Results}

\subsection{Body Weight}

Mean body weight increased over time for all groups, and no significant differences were found among the groups at any point in time (Figure 2).

\subsection{CT-Based Assessment of the Mandibular Condyle}

In the three-dimensional constructed images, both the extraction group and the powder group showed smaller mandibular condyle sizes compared to the control group 


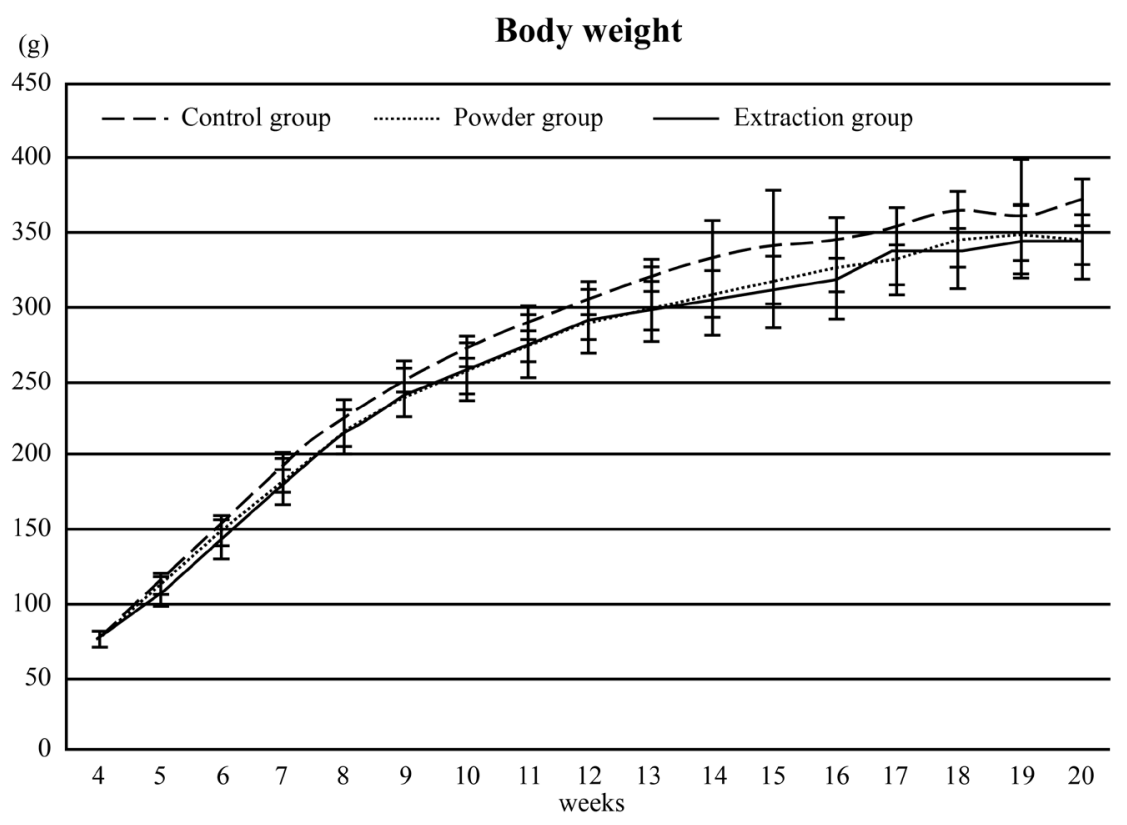

Figure 2. Rat body weight by week. Body weight increased as the rats aged, and no significant difference was found across the groups at any point in time.

(Figure 3). BV in the mandibular condyle was $0.0054 \pm 0.0003 \mathrm{~cm}^{3}$ for the extraction group, $0.0055 \pm 0.00038 \mathrm{~cm}^{3}$ for the powder group, and $0.0085 \pm 0.00041 \mathrm{~cm}^{3}$ for the control group. BMC in the mandibular condyle was $4.76 \pm 0.313 \mathrm{mg}$ for the extraction group, $4.77 \pm 0.367 \mathrm{mg}$ for the powder group, and $7.90 \pm 0.35 \mathrm{mg}$ for the control group. BMD in the mandibular condyle was $872.9 \pm 16.83 \mathrm{mg} / \mathrm{cm}^{3}$ for the extraction group, $862.2 \pm 12.49 \mathrm{mg} / \mathrm{cm}^{3}$ for the powder group, and $924 \pm 18.75 \mathrm{mg} / \mathrm{cm}^{3}$ for the control group. The extraction group and the powder group showed significantly smaller values than the control group for all of all three of these parameters (Figure 4).

\subsection{Histological Observation}

Figure 5 shows the tissue images of sagittal sections of the mandibular condyle. In the control group, the cartilage was observed to be smoothly curved so as to cover the mandibular condyle. Additionally, the cells were regularly arranged in the order of AZ, IZ, and HZ from the surface layer (Figure 5(a), Figure 5(b)). However, in two of the seven rats in the extraction group, and in one of the seven rats in the powder group, the cartilage had abnormal findings such as disruption of the arrangement of cell layers, coagulation of chondrocytes, and duplication of the tidemark (Figure 5(c)). Additionally, the cancellous bone in the extraction group showed many large osteoblasts with large numbers of nuclei (Figure 5(d)).

\subsection{Bone Histomorphometry of Cartilage}

We performed bone histomorphometry of the cartilage at the middle of a sagittal crosssection of the mandibular condyle (Figure 6). No significant differences in the areas of the chondrocyte layers were found among the groups for any layer, but individual 

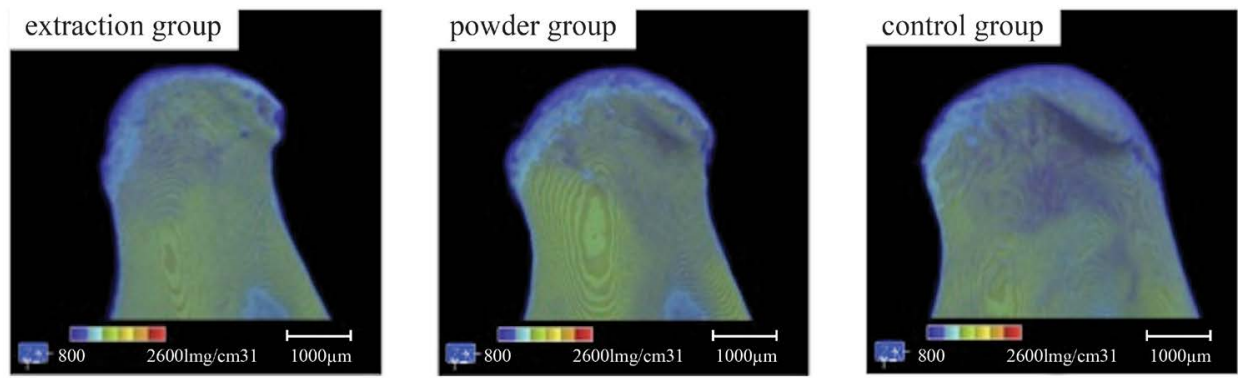

Figure 3. Three-dimensional micro-CT images. The extraction group and the powder group showed smaller mandibular condyle sizes than the control group.

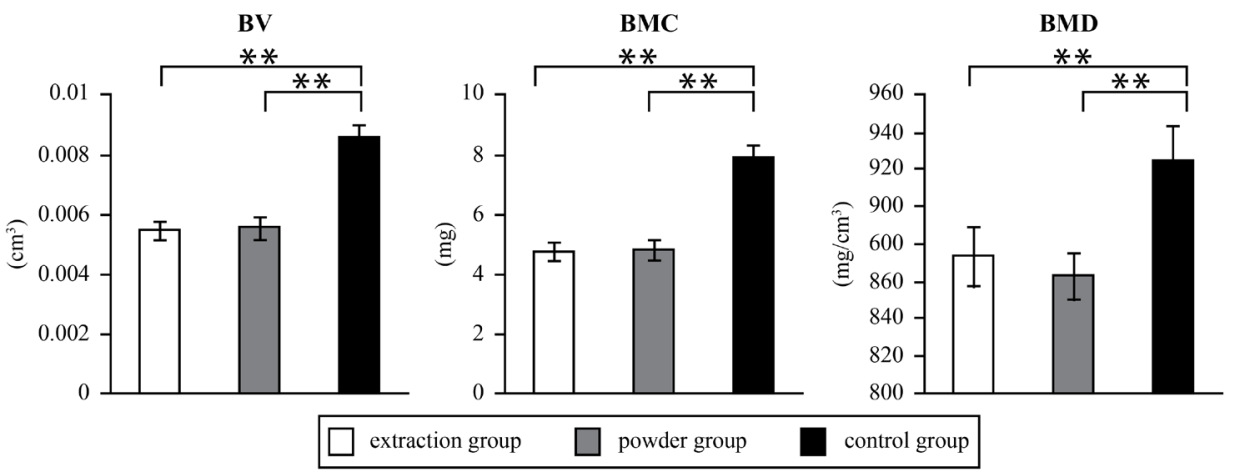

Figure 4. BV, BMC, and BMD from micro-CT analysis. In all cases, the extraction group and the powder group were significantly smaller than the control group. Data are presented as the mean \pm the standard error of the mean $\left({ }^{\star *} \mathrm{P}<0.01\right)$. BV: bone volume. BMC: bone mineral content. BMD: bone mineral density.

differences were larger within the extraction group and the powder group compared to the control group. The number of cells in the AZ was significantly higher in the extraction group compared to the powder group.

\subsection{Bone Histomorphometry of Cancellous Bone}

We performed bone histomorphometry of the secondary cancellous bone (Figure 7). $\mathrm{BV} / \mathrm{TV}$, a bone volume parameter, was significantly smaller for the extraction group $(54.47 \pm 5.83)$ and the powder group $(59.03 \pm 6.87)$ than for the control group (68.73 \pm 6.77). Tb.Th was also significantly smaller for the extraction group $(60.39 \pm 3.66)$ and the powder group $(73.75 \pm 6.68)$ than for the control group (117.20 \pm 18.19$)$. Likewise, $\mathrm{OV} / \mathrm{BV}$, an osteogenesis parameter, was significantly smaller for the extraction group $(7.12 \pm 1.15)$ and the powder group $(6.55 \pm 1.48)$ than for the control group $(8.92 \pm$ 2.47). N.Ob/BS was significantly smaller for the extraction group $(30.61 \pm 4.02)$ and the powder group $(27.02 \pm 4.86)$ than for the control group (52.87 \pm 16.02$)$. ES/BS, a bone resorption parameter, was significantly higher for the extraction group (46.54 \pm 7.28 ) and the powder group $(30.50 \pm 5.31)$ than for the control group $(10.95 \pm 1.93)$. N.Mo.Oc/BS was significantly higher for the extraction group $(1.82 \pm 0.55)$ and the powder group $(1.10 \pm 0.51)$ than for the control group $(0.73 \pm 0.45)$. N.Mu.Oc/BS was 


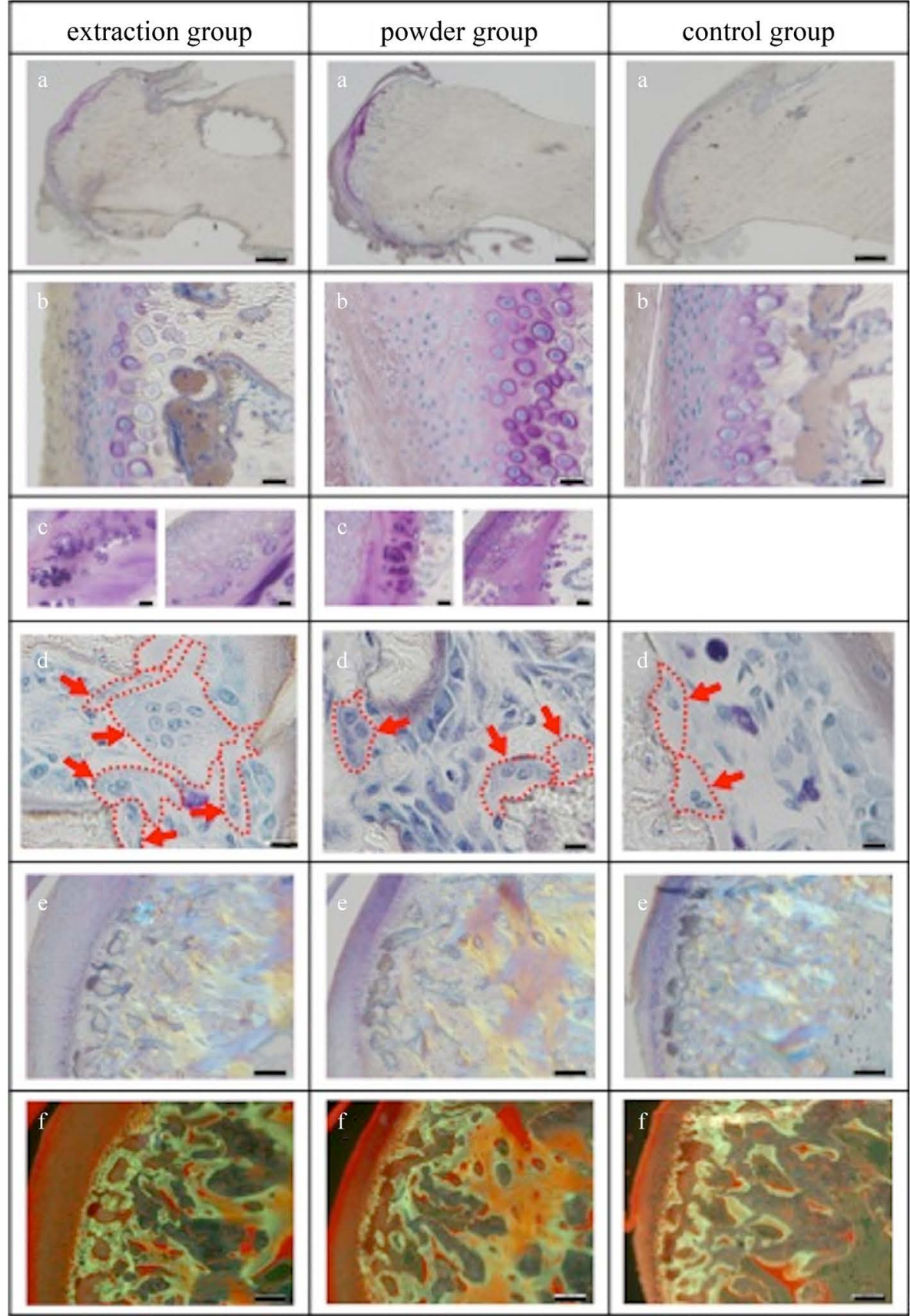

Figure 5. Tissue images of sagittal sections of the mandibular condyle under Villanueva Bone Stain; (a) Low magnification of the mandibular condyle. Bar: $500 \mu \mathrm{m}$; (b) High magnification of the cartilage of the mandibular condyle. Bar: $20 \mu \mathrm{m}$; (c) Abnormal findings in the extraction group and the powder group, including disruption of the arrangement of cell layers, coagulation of chondrocytes, and duplication of the tidemark. Bar: $20 \mu \mathrm{m}$; (d) High magnification of the secondary cancellous bone. Arrows indicate multinuclear osteoclast arrow. In the extraction group, we observed many large multinuclear osteoclasts with a large numbers of nuclei. Bar: $10 \mu \mathrm{m}$; (e) Polarizing microscopic images of the mandibular condyle. Bar: $100 \mu \mathrm{m}$; (f) Fluorescence microscopic images of the mandibular condyle. The emitted yellow and green light are tetracycline (injected seven days before imaging) and calcein (injected one day before imaging), respectively. Bar: $100 \mu \mathrm{m}$. 


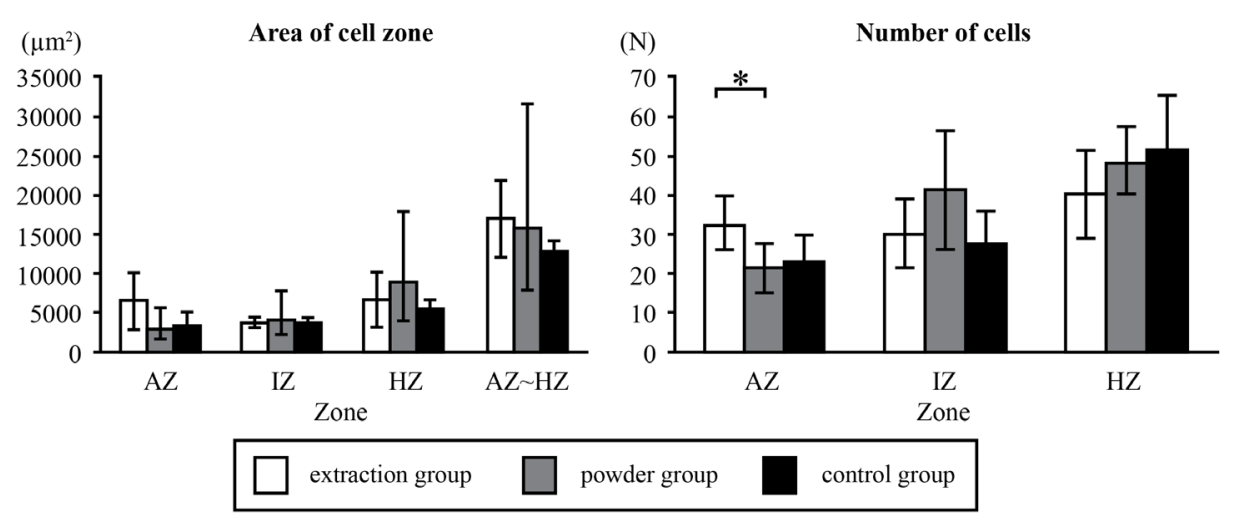

Figure 6. Bone Histomorphometry of Chondrocytes in the Central Portion of a Sagittal CrossSection of the Mandibular Condyle. No significant differences in the areas of the chondrocyte layers were found among the groups for any layer. In the AZ, the number of cells was significantly larger in the extraction group than in the powder group. Data are presented as the mean \pm the standard error of the man $\left({ }^{\star} \mathrm{P}<0.05\right)$. AZ: articular zone. IZ: intermediate zone. HZ: hypertrophic zone.
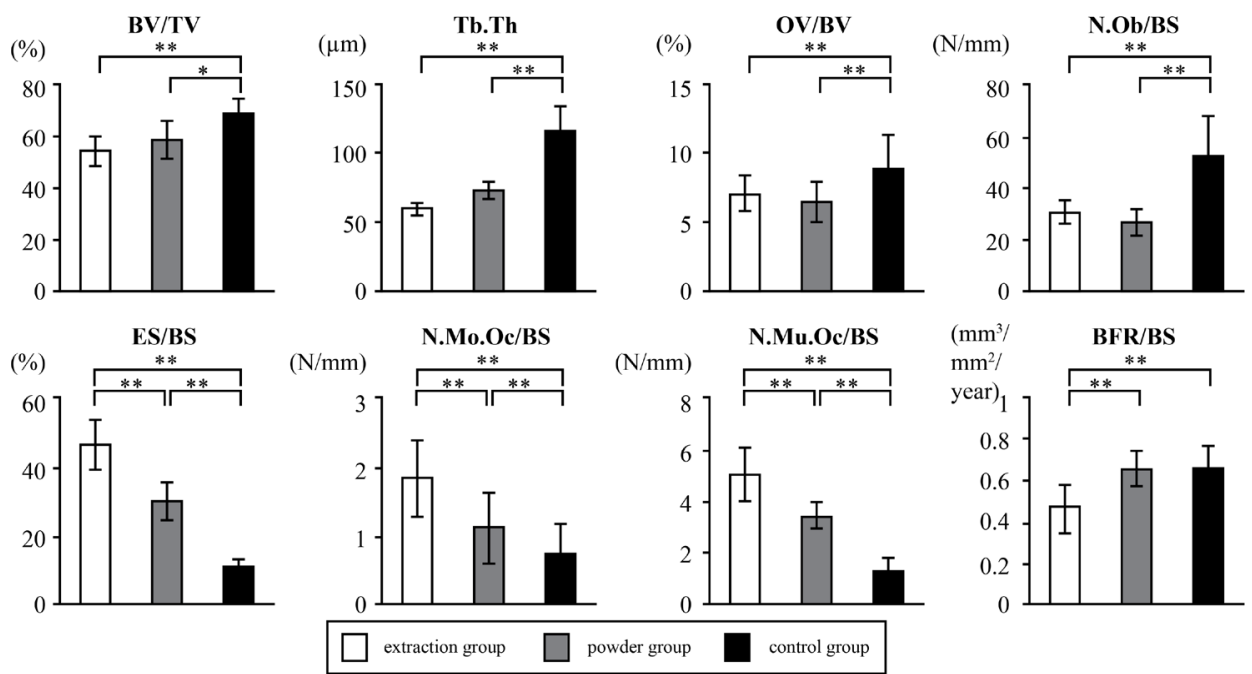

Figure 7. Histomorphometric Analysis of the Secondary Cancellous Bone in the Mandibular Condyle. BV/TV: bone volume/tissue volume. Tb.Th: trabecular thickness. OV/BV: osteoid volume/bone volume. N.Ob/BS: osteoblast number/bone surface. ES/BS: eroded surface/bone surface. N.Mo.Oc/BS: mononuclear osteoclast number/bone surface. N.Mu.Oc/BS: multinuclear osteoclast number/bone surface. BFR/BS: bone formation rate/bone surface. Data are presented as the mean \pm the standard error of the mean $\left({ }^{*} \mathrm{P}<0.05\right.$ and $\left.{ }^{* *} \mathrm{P}<0.01\right)$.

also significantly higher for the extraction group $(5.05 \pm 1.08)$ and the powder group $(3.43 \pm 0.55)$ than for the control group $(1.29 \pm 0.52)$. BFR/BS, a kinetic parameter, was significantly smaller for the extraction group $(0.47 \pm 0.11)$ than for either the powder group $(0.65 \pm 0.07)$ or the control group $(0.67 \pm 0.10)$

\section{Discussion}

The purpose of this study was to clarify the effects of long-term lack of teeth and use of 
soft food on bone turnover in the mandibular condyle. Our results suggest that lack of teeth and soft foods may cause a worsening of bone microstructure, reduced osteogenesis, and promotion of bone resorption.

Wistar rats were used for our study due to their short growth time and because of previous reports in which masticatory functional loss was induced in rats by tooth extraction and soft food [1] [5] [7] [8] [12] [13]. Similarly to induce masticatory functional loss in rats, the extraction group was fed a diet of powdered food following extraction of the maxillary molars, and a separate powder group was fed powdered feed in the absence of tooth extraction. Extractions were performed at four weeks of age, which corresponds to when the third molars erupt. We evaluated all animals at the age of twenty weeks, which is when the development of the mandibular bone has been reported to be complete [7].

No significant differences in body weight were found among all groups and all weeks. This result establishes that tooth extraction and powdered feed did not affect the general physical condition of our experimental animals. Thus, the changes in the mandibular condyle seen in our results do not appear to be caused by any change in the general physical conditions of the animals.

The extraction group and the powder group were observed to have significantly smaller BMC and BMD values than the control group by CT analysis. Our results agree with a previous study [9], which reported that experimental animals that had undergone tooth extraction and been put on a soft food diet show a decreased size of the mandibular condyle. Additionally, Itou et al. [4] reported that mice raised on powdered feed show a significant decrease in the mass of the masseter muscle despite showing no decrease in body weight. When rats are given solid feed, the feed is crunched several times for two to three seconds by the front teeth and then chewed for several seconds by the molars; when given powdered feed, they use only the molars to chew the feed for several seconds [15]. Thus, there is a clear difference in the activity of the masticatory muscle. According to a study involving humans [16], the conditions of the muscular process, the mandibular condyle, and the mandibular angle are strongly affected by the functioning of the masticatory muscles, including the temporal muscle, the masseter muscle, and the medial and lateral pterygoid muscles, and this provides the basis for a theory of the formation of the skeleton based on function. Furthermore, Mayama [17] reported a significantly lower BMC in the mandibles of patients with congenital lack of permanent teeth. This same report speculated that the reason for this BMC reduction could be a delay in the precipitation of bone mineral, a thinning of the density of the trabecula, or a reduction in masticatory activity due to an insufficient number of teeth causing a reduction in trabecula density. It was also inferred that mechanical stimulations such as biting and chewing lead to an increase in BMC. We therefore speculate that the extraction group and the powder group in our study required less masticatory activity to open and close the mouth compared to the control group, and that the corresponding reduction in activity of the temporal muscle, the masseter muscle, and the medial and lateral pterygoid muscles inhibited mandibular condyle development and 
reduced $B M C$ and $B M D$.

We observed a deformed cartilage surface in the mandibular condyle in two out of seven rats in the extraction group and in one out of seven rats in the powder group. Additionally, abnormal findings such as disruption of the arrangement of chondrocyte layers, coagulation of chondrocytes, and duplication of the tidemark were found in the cartilage of the mandibular condyle in the extraction group and the powder group. Ikeda et al. [18] reported that regressive changes of the trabecular structure can be caused by liquid feed, and these changes can reduce the resistance of the mandibular condyle to mechanical load and thereby increase the occurrence of deformation of the temporomandibular joint. Histological changes such as localized circulatory disorders in the rear portion of the articular disc of the temporomandibular joint, irregular directionality of fibers, coagulation of chondrocytes in the cartilage of the mandibular condyle, and destruction of cartilage have also been reported to be associated with onesided extraction of molars in humans and rats [19] [20]. In addition, irregularity and duplication of the tidemark, i.e., the boundary between the noncalcified surface layer and the calcified deep layer of articular cartilage, have been observed in temporomandibular arthrosis [21]. Because there were similar observations in the present study, a condition similar to temporomandibular arthrosis may exist in the cartilage of the mandibular condyle in the extraction group and the powder group. Pediatric dental clinics occasionally encounter cases of temporomandibular arthrosis accompanying mandibular condyle deformation in young patients, suggesting a relationship with the regressive changes in the mandibular condyle caused by masticatory functional loss and soft food during the growth period. Additionally, many large osteoclasts with extremely large numbers of nuclei were found in cancellous bone in the extraction group. Although mononuclear osteoclasts can resorb bone, most osteoclasts that actively resorb bone are large and multinuclear. This suggests that resorption activity was particularly high in the extraction group.

We found no significant differences in the areas of the chondrocyte layers among the groups for any layer using histomorphometry of the cartilage. However, we did observe large individual differences within the extraction group and the powder group compared to the control group. It is thought that the cartilage of the mandibular condyle is a region that is a supporting point for loads that respond to compressions, shears, and tensile stresses generated by various jaw functions [22]. Our histomorphometry results suggest that tooth extraction and powdered feed affect the mass of various chondrocyte layers.

Two bone volume parameters, BV/TV and Tb.Th, were observed to be significantly smaller for the extraction group and the powder group than for the control group by histomorphometry of the cancellous bone. Additionally, two osteogenesis parameters, $\mathrm{OV} / \mathrm{BV}$ and N.Ob/BS, were significantly smaller for the extraction group and the powder group than for the control group. EB/BS, N.Mo.Oc/BS, and N.Mu.Oc/BS, which are bone resorption parameters, were significantly higher for the extraction group and the powder group than for the control group, and the kinetic parameter 
BFR/BS was significantly smaller for the extraction group than for either the powder group or the control group. This suggests that reduced osteogenesis and strengthened bone resorption occur in secondary cancellous bone, and as a result, the rate of osteogenesis is lowered, and there is a reduction in bone volume and a thinning of the trabecula. Indeed, Wolff's rule states that "bone forms and resorbs in accordance with the dynamic environment, adjusting its shape and mass in a way that is suitable for maintaining its strength" [23]. We report that such changes appeared to have occurred in the cancellous bone in the extraction group and in the powder group due to reduced masticatory stimulation exerted on the mandibular condyle as a result of tooth extraction and powdered feed. This result is supported by the reduced BV, BMC, and BMD observed by CT analysis in the extraction group and the powder group compared to the control group. We speculate that the masticatory stimulation to the mandibular condyle was lower for the extraction group than for the powder group due to the effects of tooth extraction. However, the powder group did show CT and histomorphometrical results similar to those of the extraction group, suggesting that soft food in the absence of tooth loss causes mandibular condyle growth inhibition.

Regarding the relation between cartilage and cancellous bone, the HZ in the mandibular condyle is known to be replaced by bone [14]. This suggests that the abnormality of the cartilage in the extraction group and the powder group affected the cancellous bone. Furthermore, Kato et al. [13] reported that a liquid diet inhibits cell proliferation in the IZ in rats, which in turn leads to inhibited cartilage growth and underdevelopment of the mandibular condyle, similar to the results of this study.

\section{Conclusion}

Our results suggest that both a lack of teeth and a soft food diet inhibit the growth and development of the cartilage and cancellous bone of the mandibular condyle. Appropriate masticatory stimulation may therefore be necessary for normal formation of the mandibular condyle.

\section{Acknowledgements}

We would like to thank Dr. Akemi Ito (Ito Bone Histomorphometry Institute Co., Niigata, Japan) for her assistance with the histological analysis of cancellous bone.

\section{References}

[1] Hichijo, N., Tanaka, E., Kawai, N., van Ruijven, L.J. and Langenbach, G.E. (2015) Effects of Decreased Occlusal Loading during Growth on the Mandibular Bone Characteristics. PLoS $O N E, 10$, e0129290.. https://doi.org/10.1371/journal.pone.0129290

[2] Dias, G.J., Cook, R.B. and Mirhosseini, M. (2011) Influence of Food Consistency on Growth and Morphology of the Mandibular Condyle. Clinical Anatomy, 24, 590-598. https://doi.org/10.1002/ca.21122

[3] Katsaros, C., Berg, R. and Kiliaridis, S. (2002) Influence of Masticatory Muscle Function on Transverse Skull Dimensions in the Growing Rat. Journal of Orofacial Orthopedics, 63, 513. https://doi.org/10.1007/s00056-002-9903-0 
[4] Ito, G., Kuroe, K., Yasuda, H., Inoue, N. and Kamegai, T. (1982) Experimental Study on Jaw Reduction. The Journal of Japan Orthodontic Society, 41, 708-715. (In Japanese)

[5] Mavropoulos, A., Kiliaridis, S., Bresin, A. and Ammann P. (2004) Effect of Different Masticatory Functional and Mechanical Demands on the Structural Adaptation of the Mandibular Alveolar Bone in Young Growing rats. Bone, 35, 191-197.

https://doi.org/10.1016/j.bone.2004.03.020

[6] Enomoto, A., Watahiki, J., Yamaguchi, T., Irie, T., Tachikawa, T. and Maki, K. (2010) Effects of Mastication on Mandibular Growth Evaluated by Microcomputer Tomography. European Journal of Orthodontics, 32, 66-70. https://doi.org/10.1093/ejo/cjp060

[7] Muramatsu, H., Zhang, X. and Ogawa, K. (2012) Jawbone Morphology in Rats with Extracted Maxillary Molars Reared on Powdered Diet. International Journal of Oral-Medical Sciences, 11, 211-217. https://doi.org/10.5466/ijoms.11.211

[8] Abo, N., Ogawa, K. and Shimizu, K. (2013) Craniofacial Development in Rats Fed on Powdered Diet Following Extraction of All Upper Molars. International Journal of Oral-Medical Sciences, 12, 129-140. https://doi.org/10.5466/ijoms.12.129

[9] Chen, J., Sobue, T., Utreja, A., Kalajzic, Z., Xu, M., Kilts, T., Young, M. and Wadhwa, S. (2011) Sex Differences in Chondrocyte Maturation in the Mandibular Condyle from a Decreased Occlusal Loading Model. Calcified Tissue International, 89, 123-129. https://doi.org/10.1007/s00223-011-9498-9

[10] Vaid, L.K., Pradhan, P. and Chakrabarti, S. (2002) Effect of Dietary Consistency on the Growth of the Condylar Cartilage of the Mandible in Rats. Journal of the Anatomical Society of India, 51, 229-231.

[11] Kiliaridis, S., Thilander, B., Kjellberg, H., Topouzelis, N. and Zafiriadis, A. (1999) Effect of Low Masticatory Function on Condylar Growth: A Morphometric Study in the Rat. American Journal of Orthodontics and Dentofacial Orthopedics, 116, 121-125. https://doi.org/10.1016/S0889-5406(99)70207-6

[12] Pirttiniemi, P., Kantomaa, T. and Sorsa, T. (2004) Effect of Decreased Loading on the Metabolic Activity of the Mandibular Condylar Cartilage in the Rat. European Journal of Orthodontics, 26, 1-5. https://doi.org/10.1093/ejo/26.1.1

[13] Kato, T., Takahashi, S. and Domon, T. (2015) Effects of a Liquid Diet on the Temporomandibular Joint of Growing Rats. Medical Principles and Practice, 24, 257-262. https://doi.org/10.1159/000370072

[14] Blackwood, H.J.J. (1966) Growth of the Mandibular Condyle of Rats Studied with Tritiated Thymidine. Archives of Oral Biology, 11, 493-496. https://doi.org/10.1016/0003-9969(66)90155-5

[15] Yamada, G. (1992) Effects of Masseter Muscle Development and the Form of the Mandible in Developing Rats Fed a Powder Diet. Journal of Gifu Dental Society, 19, 284-230. (In Japanese)

[16] Moss, M.L. and Meehan, M. (1970) Functional Cranial Analysis of the Coronoid Process in the Rat. Acta Anatomica, 77, 11-24. https://doi.org/10.1159/000143524

[17] Mayama, H. (2004) A Study of Mandibular Bone Mineral Content and Bone Age in Young Patients with Congenitally Missing Permanent Teeth. Dentistry in Japan, 40, 71-75.

[18] Ikeda, Y., Yonemitsu, I., Takei, M., Shibata, S. and Ono, T. (2014) Mechanical Loading Leads to Osteoarthritis-Like Changes in the Hypofunctional Temporomandibular Joint in Rats. Archives of Oral Biology, 59, 1368-1376. https://doi.org/10.1016/j.archoralbio.2014.08.010

[19] Granados, J.I. (1979) The Influence of the Loss of Teeth and Attrition on the Articular 
Eminence. Journal of Prosthetic Dentistry, 42, 629-635.

https://doi.org/10.1016/0022-3913(79)90333-0

[20] Christensen, L.V. and Ziebert, G.J. (1986) Effect of Experimental Loss of Teeth on the Temporomandibular Joint. Journal of Oral Rehabilitation, 13, 587-598.

https://doi.org/10.1111/j.1365-2842.1986.tb00682.x

[21] Havelka, S., Horn, V., Spohrová, D. and Valouch, P. (1984) The Calcified-Noncalcified Cartilage Interface: The Tidemark. Acta Biologica Hungarica, 35, 271-279.

[22] MuKay, G., Yemm, R. and Cadden, S.W. (1992) The Structure and Function of the Temporomandibular Joint. British Dental Journal, 173, 127-132. https://doi.org/10.1038/sj.bdj.4807966

[23] Frost, H.M. (1994) Wolff's Law and Structural Adaptations of Bone to Mechanical Usage: An Overview for Clinicians. The Angle Orthodontist, 64, 175-188.

\section{Submit or recommend next manuscript to SCIRP and we will provide best service} for you:

Accepting pre-submission inquiries through Email, Facebook, LinkedIn, Twitter, etc.

A wide selection of journals (inclusive of 9 subjects, more than 200 journals)

Providing 24-hour high-quality service

User-friendly online submission system

Fair and swift peer-review system

Efficient typesetting and proofreading procedure

Display of the result of downloads and visits, as well as the number of cited articles

Maximum dissemination of your research work

Submit your manuscript at: http://papersubmission.scirp.org/

Or contact ojst@scirp.org 